\title{
Class V Surface
}

National Cancer Institute

\section{Source}

National Cancer Institute. Class V Surface. NCI Thesaurus. Code C60748.

The gingival margins of the tooth surfaces. 